\title{
ОБЗОР СОВРЕМЕННЫХ МЕТОДОВ ЭКОЛОГО-ЛАНДШАФТНЫХ ИСЛЕДОВАНИЙ ПРИБЕЖНЫХ АКВАТОРИЙ
}

\author{
Пьянов А. А. \\ Тихоокеанский институт географии ДВО РАН, Владивосток \\ Pyanov_a@mail.ru
}

Аннотация. В статье приведен обзор существующих и ныне использующихся методов, которые позволяют проводить исследования прибрежных акваторий, применительно к широкому кругу задач, а именно: экологических, геоморфологических, марикультурных, промышленных, общехозяйственных и т.д. Среди обозреваемых методов выделяются прямые наблюдения и анализ информации, полученной удалённо. Для принятия эффективных мер по улучшению экологической ситуации необходимо комплексное изучение основных факторов, определяющих динамику абиотической составляющей экосистемы, анализ ключевых механизмов самоочищения, скопления массовых видов продуцентов, а также разработка эффективной системы мониторинга состояния акватории. Помимо классического прямого водолазного исследования с помощью легководолазного снаряжения (SCUBA) рассматриваются методы исследования с использованием дистанционных телеуправляемых необитаемых подводных аппаратов (ТНПА), а также анализ спутниковых снимков и обследование посредством кабельной камеры. Актуальность подводных исследований морских ландшафтов обусловлена всё более нарастающим антропогенным воздействием на природную среду и необходимостью поиска максимально эффективного способа ведения природопользовательской деятельности, в том числе и в переходных геосистемах, например, на стыке наземной и водной среды. На данный момент вполне известным и обоснованным является тот факт, что максимальная продуктивность экосистемы наблюдается в прибрежной зоне морей и океанов, следовательно, и основная природопользовательская деятельность ведётся именно там. Подробное изучение, а также описание подводных ландшафтов является ключом к ведению рационального природопользования в морской среде. Долговременные наблюдения с помощью удалённо расположенных приборов позволяют накапливать и анализировать данные за десятилетия наблюдений и на основании их строить модели и прогнозы, связанные с будущими потенциальными опасностями природного характера, такими как цунами, «красные приливы», ураганы, изменения русел рек и иных событий, влияющих на экосистемы. Результаты комплексных исследований подводных ландшафтов с применением всех методов, доступных исследователям, являются наиболее достоверными.

Ключевые слова: ландшафтные исследования, подводные аппараты, водолазные работы, подводные камеры, исследования подводных ландшафтов, спутниковые методы исследования. 


\title{
OVERVIEW OF MODERN METHODS OF ECOLOGICAL-LANDSCAPE RESEARCH OF COASTAL WATERS
}

\author{
Pyanov A.A \\ Pacific Geographical Institute FEB RAS, Vladivostok
}

\begin{abstract}
Annotation. This article provides an overview of existing and currently used methods that allow for the study of coastal waters, with reference to a wide range of tasks, namely: environmental, geomorphological, maricultural, industrial, general economic, etc. Among the observed methods, direct observations and analysis of information obtained remotely are distinguished. Among the observed methods, direct observations and analysis of information obtained remotely are distinguished. To take effective measures to improve the ecological situation, a comprehensive study of the main factors determining, first of all, the dynamics of the abiotic component of the ecosystem, the analysis of key self-cleaning mechanisms, the accumulation of mass species of producers, and the development of an effective system for monitoring the state of the water area are necessary. In addition to the classic direct diving research using light diving equipment (SCUBA), research methods using remote telecontrolled uninhabited underwater vehicles (ROV), as well as analysis of satellite images and examination using a cable camera are considered. The relevance of underwater studies of marine landscapes is due to the increasing anthropogenic impact on the natural environment and the need to find the most effective way to conduct nature management activities, including in transitional geosystems, for example, at the junction of terrestrial and aquatic environments. The relevance of underwater studies of marine landscapes is due to the increasing anthropogenic impact on the natural environment and the need to find the most effective way to conduct nature management activities, including in transitional geosystems, for example, at the junction of terrestrial and aquatic environments. At the moment, the fact that the maximum productivity of the ecosystem is observed in the coastal zone of the seas and oceans is quite well-known and justified, therefore, the main nature-user activity is carried out there. A detailed study, as well as a description of underwater landscapes, is the key to rational nature management in the marine environment. Long-term observations using remotely located instruments make it possible to accumulate and analyze data from decades of observations and, based on them, build models and forecasts related to future potential natural hazards, such as tsunamis, red tides, hurricanes, changes in river channels and other events, affecting ecosystems. The results of comprehensive studies of underwater landscapes are the most reliable, only using all methods available to researchers.
\end{abstract}

Key words: landscape studies, underwater vehicles, diving works, underwater cameras, research of underwater objects, satellite research methods.

Актуальность подводных исследований морских ландшафтов обусловлена всё более нарастающим антропогенным воздействием на природную среду и необходимостью поиска максимально эффек- 
тивного способа ведения природопользовательской деятельности, в том числе и в переходных геосистемах, например, на стыке наземной и водной среды. На данный момент вполне известным и обоснованным является тот факт, что максимальная продуктивность экосистемы наблюдается в прибрежной зоне морей и океанов, следовательно, и основная природопользовательская деятельность ведётся именно там. Подробное изучение и описание подводных ландшафтов является ключом к ведению рационального природопользования в морской среде.

Классическим, наиболее распространённым и универсальным методом исследования подводных ландшафтов является водолазная съёмка с использованием легководолазного снаряжения. По своей природе подводные ландшафты располагаются в чуждой и чаще всего враждебной для человека среде, и они не могут быть описаны, изучены и закартированы без специальных приборов, инструментов и снаряжения [1]. Для удобного изучения подводных ландшафтов с помощью легководолазного снаряжения (гидрокостюм сухого или мокрого типа, акваланг и сопутствующее оборудование), крайне полезным оказывается изготовление «приборного узла», который состоит из пластикового планшета, на котором закрепляется компас, простой карандаш, линейка и глубиномер. При осуществлении водолазных спусков необходима географическая привязка, для этого вполне подходят бытовые GPS-приёмники типа Garmin. Начиная с середины 2010-х годов широкое распространение получили компактные экшн-камеры типа GoPro, SJCam и им подобные. Камера закрепляется на пластиковом планшете, либо на маске водолаза. В последствии разбор видеоматериала с камеры позволяет более подробно обозначить важные детали водолазного разреза, а если разрезов в течение дня было много, то камера становится незаменимым помощником.

Работы по картографированию удобнее всего проводить с надувной лодки (RIB) или лёгкого катера, поскольку иногда приходится выполнять работы на глубинах около 1 метра. На плавательное средство необходимо закрепить эхолот и выставить на карт-плоттере режим записи, через 3 или 5 секунд. Так же крайне полезным для экономии времени оказывается нанесение «сетки станций» на карту, по которым в дальнейшем будет осуществляться работа (для этого используется программное обеспечение, прилагаемое к эхолотам Garmin, Map source либо Base Camp, либо программа Google Earth). На выходе 
получается массив данных с информацией о глубинах на данной акватории. Для наиболее корректного отображения реальной ситуации необходимо пройти по бухте или иной обследуемой акватории галсами, чтобы покрыть весь возможный диапазон глубин. Затем по данным, полученным с помощью водолазного обследования, камер или ТНПА (телеуправляемый необитаемый подводный аппарат) осуществляется построение карты грунтов, затем, используя программный пакет Surfer (Golden software coorp.), карта глубин совмещается с картой грунтов для получения ЦМР (цифровой модели рельефа). При необходимости нанесения на карту скоплений животных или растений необходимо рассчитать их среднюю плотность на каждом водолазном разрезе и, используя географическую привязку, нанести на карту.

Если по какой-то причине отсутствует возможность или целесообразность в непосредственном нахождении человека под водой, то можно прибегнуть к иным методам обследования морского дна. Самый простой и относительно дешёвый метод - это аналоговая (или цифровая) кабельная камера (рис. 1), видеовыход которой подключен к экрану для вывода данных. Некоторым затруднением при использовании данной техники может являться вопрос подачи питания, но опыт показывает, что наиболее рациональным решением является использование автомобильного аккумулятора с напряжением $12 \mathrm{v}$ и ёмкостью 50-60 ампер\час. Такого источника энергии с запасом хватает на неделю ежедневной работы в море. Кабельная камера опускается в воду и по данным, выводимым на экран, осуществляется описание подводного ландшафта. Наиболее удобной является работа в паре, где один опускает и поднимает камеру, а второй анализирует видеоданные и делает записи в журнале. Одним из недостатков этого метода является отсутствие возможности записывать видео с камеры (недостаток актуален для аналоговых камер, в цифровых моделях этот вопрос решён).

Телеуправляемый необитаемый подводный аппарат (ТНПА) аппарат, оснащенный специальным оборудованием, погружаемый в воду и управляемый с поверхности пилотом или группой операторов (пилот, навигатор и др.). Подводное обследование с помощью ТНПА позволяет получить объективную визуальную информацию о состоянии подводных объектов в режиме реального времени с возможностью записи с целью последующего детального анализа. Основной взор конструкторов ТНПА на данный момент направлен на созда- 
ние глубоководных моделей, в то время как лёгкие и компактные аппараты остаются менее востребованными и зачастую производятся небольшими партиями под конкретные цели и заказчиков, а иногда компании-производители вынуждены обращаться к краудфайндинговым платформам для сбора средств на производство.

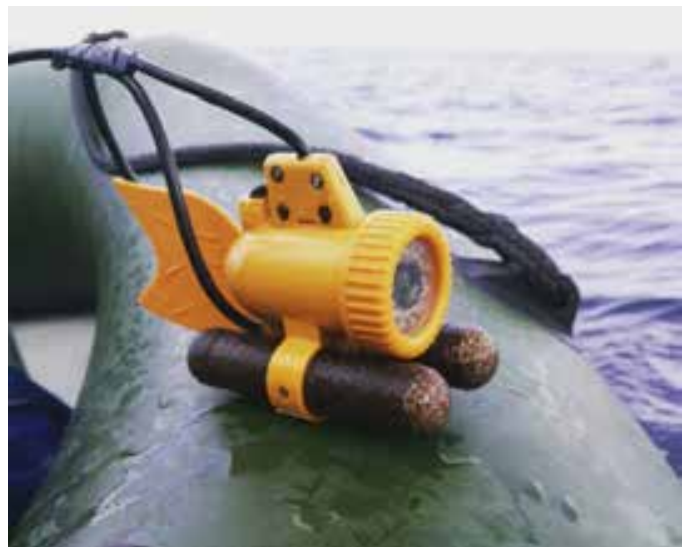

Рис. 1. Кабельная камера BEST WILL Крупные аппараты, такие как Sub-fighter 10k (или старшие модели Sub-fighter) или JASON/MEDEA (ROV) (рис. 2) преимущественно используются на больших глубинах, при исследовании абиссальных равнин (глубины около 5-6 км). Работа на таких аппаратах сопряжена со сложной логистикой, дороговизной приборов и наличием высококвалифицированного персонала, способного с борта судна управлять подводным устройством. В то же время, примерно с 2015 года, вслед за широким развитием квадрокоптеров (дистанционно управляемых воздушных дронов гражданского назначения) стали появляться и подводные дроны. Главными преимуществами такой техники явля-

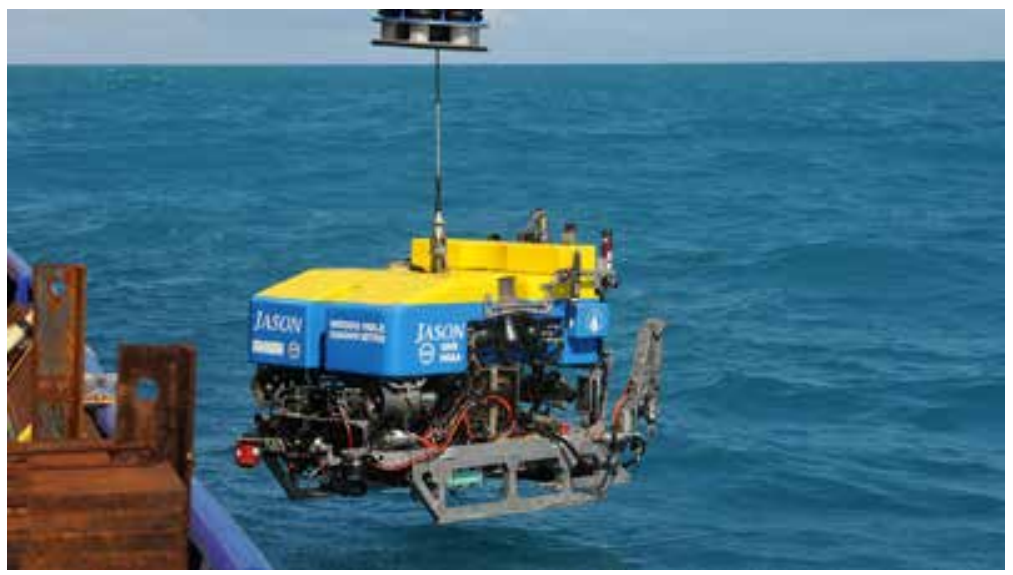

Рис. 2. Телеуправляемый необитаемый подводный аппарат JASON/MEDEA 
ется относительно невысокая стоимость (в среднем около $2500 \$$ и меньше), лёгкость, простота настройки (для управления подводным дроном необходим смартфон с операционной системой Android либо iOS). В качестве примера можно привести подводный дрон от компании Chasing innovation, Gladius Advanced pro.

Дрон Gladius и большинство ему подобных имеют 4 двигателя, камеру с разрешением $4 \mathrm{k}$ и аккумуляторную батарею ёмкостью около 5000-6000 mAh. Для связи смартфона с аппаратом используется смартфон с предустановленным программным обеспечением, на который выводится изображение с камеры подводного аппарата. В силу того, что на данный момент технологии не позволяют установить беспроводной канал связи непосредственно между аппаратом, находящимся под водой, и смартфоном, используется кабельная станция, которая представляет из себя плавающий буй с Wi-fi приёмником и кабелем, подсоединённым к подводному дрону. Важной деталью при обследовании является возможность установить курс, по которому необходимо вести аппарат, не сбиваясь с него. В подводных дронах, как правило, имеется цифровой компас, гироскоп, а на управляющем буе находится GPS-передатчик. Такой комплекс приборов при должной настройке и калибровке позволяет достаточно чётко привязывать маршрут аппарата.

Из недостатков можно выделить ограниченную дистанцию действия дрона (от 50 до 100 метров кабеля) и необходимость регулировки нейтральной плавучести (штатная грузовая система может быть выверена не совсем корректно, дрон будет иметь положительную или отрицательную плавучесть). Слабым местом в конструкции является способ крепления шнура к аппарату. В остальном управление таким аппаратом достаточно легко осваивается и в дальнейшем навык быстро растёт.

Помимо неуправляемых аппаратов, существуют в основном «бумажные проекты» автомобилей-амфибий и прочих устройств для мониторинга морской среды [2].

Кроме прямых методов исследования прибрежных акваторий во второй половине XX века в связи с запуском гражданских спутников и спутниковых группировок, оснащённых приборами для съёмки данных о поверхности земли или воды, появилась возможность использования дистанционных данных. Первым спутником, запущенным в 1972 году, был LANDSAT 1. 
- Landsat 1 (изначально ERTS-1, Earth Resources Technology Satellite 1) - запущен 23 июля 1972, прекратил работу 6 января 1978

- Landsat 2 (ERTS-B) - запущен 22 января 1975, прекратил работу 22 января 1981

- Landsat 3 - запущен 5 марта 1978, прекратил работу 31 марта 1983

- Landsat 4 - запущен 16 июля 1982, прекратил работу в 1993

- Landsat 5 - запущен 1 марта 1984, прекратил работу 21 декабря 2012

- Landsat 6 - запуск 5 октября 1993, на целевую орбиту не выведен

- Landsat 7 - запущен 15 апреля 1999, функционирует. В мае 2003 произошел сбой модуля Scan Line Corrector (SLC). C сентября 2003 используется в режиме без коррекции линий сканирования, что уменьшает количество получаемой информации до 75 \% от изначальной.

- Landsat 8 - запущен 11 февраля 2013. 30 мая 2013 после окончания тестирования и настройки передан под управление USGS [3].

В дальнейшем появились и иные спутниковые группировки, среди которых есть и коммерческие аппараты с высокой степенью разрешения, например, IKONOS (разрешающая способность до 1 метра). Важнейшим этапом в этой области был запуск аппарата Teppa (EOS AM-1).

Терра несёт на борту следующие пять дистанционных зондов для наблюдения за окружающей средой и изменениями климата.

- ASTER - Японский зонд, фотографирующий Землю с высоким разрешением в 15 диапазонах электромагнитного спектра, от видимого до инфракрасного излучения. С разрешением от 15 до 90 метров, изображения ASTER используются для создания подробных карт температуры поверхности Земли, излучательной способности (emissivity), отражательной способности и высоты.

- CERES - радиометр.

- MISR - 9 цифровых фотоаппаратов, приспособленных для измерения солнечного излучения, отражаемого Землёй (как поверхностью, так и атмосферой) в различных направлениях и диапазонах спектра.

- MODIS - фотографирует в 36 диапазонах спектра, с длиной волны от 0,4 мкм до 14,4 мкм и разрешением от 250 м до 1 км. Пред- 
назначен для наблюдения за глобальной динамикой планеты Земли (изменения облачности, радиационный баланс и процессы, происходящие в океанах, на суше, и в нижних слоях атмосферы.

- MOPITT - наблюдает за характером загрязнения атмосферы [4].

Среди основных параметров, которые анализируются и изучаются по спутниковым данным можно выделить: температуру и солёность, течения, уровень моря, состояние поверхностного слоя и гидродинамику, биологическую продуктивность поверхностного слоя, рельеф мелководий, зонирование биотопов.

Данные со спутника помогают учёным понимать распространение загрязнения по всей планете. Инструменты Терра были использованы в научных работах, исследующих тенденции в глобальном загрязнении угарным газом и аэрозолем. Собранные спутником данные, станут в конечном счёте новым, 15-летним глобальным банком данных. Современные приборы, установленные на спутниках, позволяют идентифицировать разные типы поверхностей, данная методика основана на разности в коэффициенте отражения. Разные типы поверхностей по-разному отражают электромагнитное излучение прибора, следовательно, возможно зонирование разных областей, но для высокой достоверности необходима «полевая заверка» специалистами и уточнение границ различных выделов. Долговременные наблюдения с помощью удалённо расположенных приборов позволяют накапливать и анализировать данные за десятилетия наблюдений и на основании их строить модели и прогнозы, связанные с будущими потенциальными опасностями природного характера, такими как цунами, «красные приливы», ураганы, изменения русел рек и иных событий, влияющих на экосистемы. Наиболее достоверными данными о подводных ландшафтах являются результаты комплексных исследований, полученные с применением всех методов, доступных исследователям.

В заключении следует отметить, что ни один из приведённых методов исследования не является универсальным.

Научный руководитель: в.н.с. ТИГ ДВО РАН, к.г.н. Жариков В.В.

\section{Литература}

1. Преображенский Б.В., Жариков В.В., Дубейковский Л.В. Основы подводного ландшафтоведения. Управление морскими экосистемами. Владивосток: Дальнаука, 2000. $351 \mathrm{c}$. 
2. Семёнов А.Г. Амфибия с солнечной батареей // Транспорт. Транспортные сооружения. Экология. 2016. № 2. С. 100-117.

3. Landsat Science. History [Электронный ресурс]. - Режим доступа: https://landsat.gsfc.nasa.gov/about/history/ (дата обращения: 12.08.2019).

4. NASA's Goddard Space Flight Center [Электронный ресурс]. - Режим доступа URL: https://www.nasa.gov/goddard (дата обращения: 12.08. 2019).

\section{References}

1. Preobrazhensky B.V., Zharikov V.V., Dubeykovsky L.V. Osnovy podvodnogo landshaftovedeniya. Upravlenie morskimi ekosistemami [Basics of underwater landscape science. Marine ecosystem management]. Vladivostok, Dalnauka Publ., 2000. 351 p.

2. Semenov A.G. Amfibiya s solnechnoj batareej [Amphibia with a sun battery]. Transport. Transport facilities. Ecology - Transport. Transportnye sooruzheniya. Ekologiya, 2016, no. 2, pp. 100-117.

3. Landsat Science. History. Available at: https://landsat.gsfc.nasa.gov/about/history/ (accessed: 12.08.2019).

4. NASA's Goddard Space Flight Center. Available at: https://www.nasa.gov/goddard (accessed: 12.08.2019). 